\title{
Investigation of the Surface Properties of Diamond-Like Carbon Films Using Multifractal Analysis
}

\author{
Narek Margaryan \\ Department of Physics, National Polytechnic University of Armenia, Yerevan 0089-0009, Armenia
}

\begin{abstract}
In this paper surface potential of DLC (diamond-like carbon) nanostructured film is studied. Fractal form of the surface of this film is discussed. Fractal parameters and surface potential as a function of them are calculated theoretically. Then obtained results are compared with the results measured by Kelvin probe method. The evolution of the surface topology and related surface potential during the deposition time is studied too. The dependence of multifractal parameters on the deposition duration was revealed.
\end{abstract}

Key words: Nanostructured film, multifractal, atom force microscopy, surface potential.

\section{Nomenclature}

$\begin{array}{ll}\text { DLC: } & \text { Diamond-like carbon } \\ \text { CVD: } & \text { Chemical vapor deposition } \\ \text { AFM: } & \text { Atom force microscopy } \\ \text { Ge: } & \text { Germanium }\end{array}$

\section{Greek letters}

$\begin{array}{ll}\alpha: & \text { specific surface energy } \\ \rho: & \text { density } \\ \mu: & \text { chemical potential }\end{array}$

\section{Introduction}

The process of forming the semiconductor layers for modern electronic devices is accompanied by a process of self-organization at the nano and micro levels. A promising approach to describe such self-similar state to the surface is the multifractal analysis [1-3]. Introduced by Benoit Mandelbrot, general pattern of geometric properties of the physical world, which is manifested in the self-similarity of its structure, has found numerous applications in materials science and has provided new opportunities for describing disordered materials microstructures using rigorous quantitative terms, such as fractal dimension. To date, it has been found that to describe the self-similarity of

Corresponding author: Narek Margaryan, Ph.D., research fields: semiconductor phsics, nanotechnology, carbón films, quantum physics. natural materials is not enough just to use one of the fractal dimensions, and the works of different authors [1-7] show that the multifractal formalism provides such an opportunity. One of the important physical parameters of the nanostructured films is surface potential, which we can calculate using the results obtained by multifractal analysis. These results allow us to propose a mathematical expression that gives an opportunity to evaluate the surface potential of the film, due to the presence of fractal structure on its surface. The methodology of multifractal parameterization of structures of materials is based on the fundamental principles of self-similarity, stochastic and fractal broken symmetry structures of natural materials [4-6]. It uses a set of self-similar measures in Euclidean space, so the characteristics of the material can be described more accurately [7-9]. The property of self-similarity is quantitatively expressed using the concept of fractal dimension. To determine the fractal dimension of the fractal and other parameters, we used obtained atomic force microscopy images for chemical vapor deposited DLC nanostructured films.

\section{Theory}

\subsection{Multifractal Analysis}

In materials science and allied areas in the majority 
of cases studied structure is presented in the form of flat images. In the modern computer technology and bitmap processing programs such flat images presented in the so-called digital (digitized) form- $-\mathrm{a}$ matrix of discrete elements of the same size images-pixels. Each pixel is attributed to three numerical characteristics (coordinates): two of them ( $i$, $j$ ) define the position of the pixel on the image plane $(x, y)$, third characteristic defines its color $z(i, j)$. The color characteristics of pixels are given by integers. Coordinates of the pixels on the plane are numbers of rows and columns of pixels in the matrix (by which the image represented in digital form) and also are a set of integers. Thus, using the color characteristics of pixels as the usual number, we can imagine a flat image of the surface topography in three dimensions [3, 7, 10-15]. Examples of such structures may be three-dimensional digital images of the surface of materials obtained by AFM (atomic force microscope) (Fig.1).

Generated by the color characteristic or height $z(i, j)$ measure

$$
\lambda_{0 i j}=\frac{z(i, j)}{\left(\sum_{i j}(z(i, j))\right.}
$$

The set of elementary cells - pixels can be directly used for multifractal analysis of images. The sum of two indices for all the pixels on a square matrix $(i, j)$ can be represented as the sum of one index $i$, if renumber the pixels using one index.

Thus, when the partition of space covering, which contains the object being studied, it is possible to generate a measure $\left\{\lambda_{i}\right\},\left(\sum_{i=1}^{N} \lambda_{i}=1\right)$-some effective distribution does not change its sign value. For anyway constructed (generalized) measure $P_{i}$ the generating function is constructed:

$$
\Gamma(q, \tau, l)=\sum_{i=1}^{N} \frac{\left(p_{i}\right)^{q}}{\left(l_{i}\right)^{\tau}}
$$

where, the summation is only for not empty cells measure $p_{i}>0, q$ and $\tau$ are arbitrary real numbers $(-\infty \leq q, \tau \leq \infty)$.
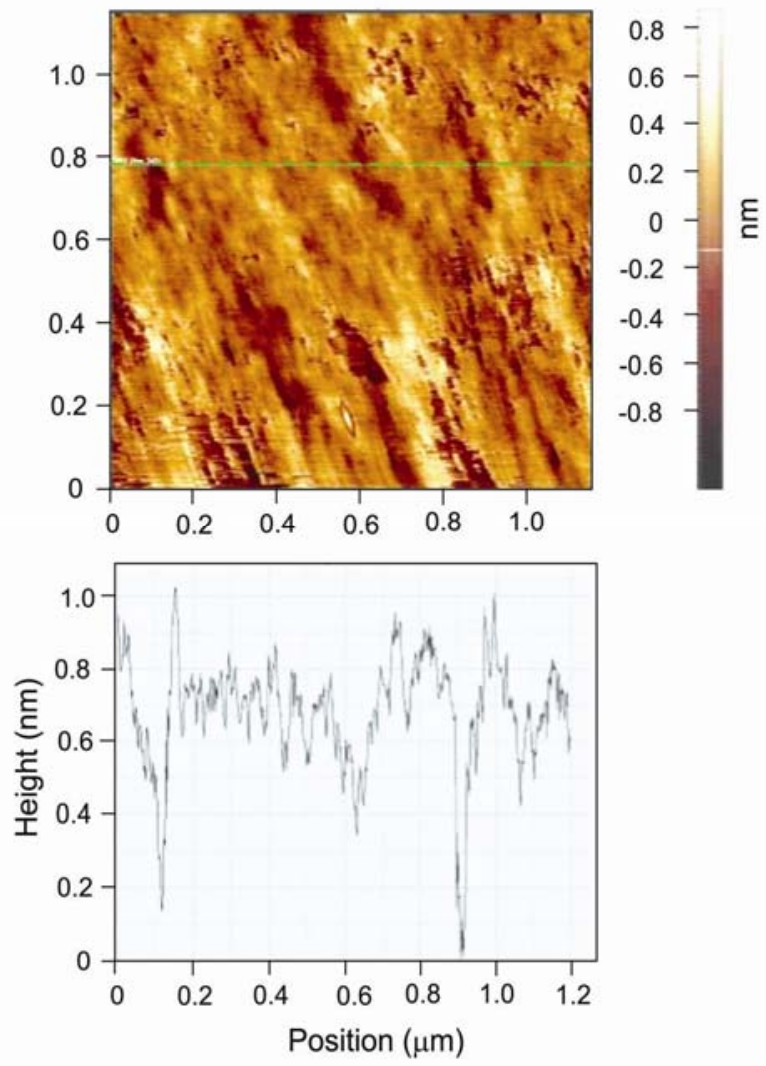

Fig. 1 AFM image of a sample of DLC grown on Ge substrate (upper panel) and cross sectional profile of thickness across the green line on the image (lower panel).

In the case of the singular behavior of measure, it takes place in many situations [16],

$$
p_{i} \cong\left(l_{i}\right)^{\alpha}, l \rightarrow 0,
$$

where, $\alpha$ is positive real number, it is easy to prove that there is a unique function of $\tau(q)$, in the limit $l \rightarrow 0$, such that $\Gamma(q, \tau(q), l \rightarrow 0)$ is finite.

For generalized correlation function $\chi$ and its exponent $\tau$ we have

$$
\begin{gathered}
\chi(q)=\sum_{i=1}^{N}\left(p_{i}\right)^{q} \sim l^{\tau(q)}, l \rightarrow 0 \\
\tau(q)=\lim _{l \rightarrow 0} \cdot \frac{\ln (\chi(q))}{\ln (l)}
\end{gathered}
$$

By summing over the cells by Eq. (4) we can get the integral form of $\chi(q)$

$$
\chi(q)=\int d \alpha \cdot p(\alpha) \cdot l^{-f(\alpha)} \cdot l^{q \alpha}
$$


where, $\rho\left(\alpha^{\prime}\right) l^{-f\left(\alpha^{\prime}\right)} d \alpha^{\prime}$ is the probability that a randomly selected $\alpha$ singularity in Eq. (3) for $p_{i}$ has a value in the range $\left(\alpha^{\prime}, \alpha^{\prime}+d \alpha^{\prime}\right)$, and $\rho(\alpha)$ is not singular function. In this way, studied self-similar set is modeled by a set of interpenetrating set of singular $\alpha$, each of which has a corresponding fractal dimension $\mathrm{f}(\alpha)$. In the $l \rightarrow 0$ limit the integral Eq. (6) is determined by some $\alpha$, at which $q \alpha-\mathrm{f}(\alpha)=\min$, obtained from the relationship between the function of $\tau(q)$ and the spectrum of singularities $\mathrm{f}(\alpha)$ :

$$
\tau(q)=q \alpha-f(\alpha), \alpha=\frac{d \tau}{d q}, q=\frac{d f}{d \alpha}
$$

From the last relations seen, the normalization condition is this $\tau(1)=0$. This fact can be expressed explicitly, putting

$$
\tau(q)=(q-1) D_{q} \text { or } D_{q}=\frac{\tau(q)}{q-1}
$$

where, $D_{q}$ is generalized Renyi dimension, at that $D_{0} \geq D_{1} \geq D_{2}$. Here $D_{0}, D_{1}$ and $D_{2}$ are fractal $(q=0)$, informative $(q=1)$ and correlative $(q=2)$ dimensions, respectively.

In the study of multifractal properties of regular fractal structures acts as $D_{0}$ fractal dimension. Strictly speaking, the term "dimension" is only applicable to the $D_{0}$, as the value of $q=0$ removes the distinction of cells of different measures, and we have to deal with the media configuration "in its purest form".

\subsection{Surface Energy Calculation}

Excess component of free energy of system, associated with the appearance of the interfaces, can be calculated from the expression

$$
d G_{S}^{e x}=d A_{s}=\alpha d S
$$

where, $d G_{S}^{e x}$ is the change in free excess Gibbs energy, due to the emerging of separation boundary, $d A_{s}$ is the work on the formation of the section area of the surface $d S, \alpha$ is the specific surface energy of the separation boundary. In this case, the component of the excess chemical potential, associated with the emergence of new geometric shapes on the surface, can be calculated by the classical expression of thermodynamics:

$$
\mu_{s}=\frac{d G_{s}}{d n}=\frac{\alpha M}{\rho} \cdot \frac{d S}{d V}
$$

where, $\rho$ and $M$ are density and molecular weight of solid phase, $d V$ and $d n$ are changes of the volume and the number of moles of the solid phase, providing change of surface of the section with area $d S$.

To formally obtain the desired expression for the surface energy associated with the fractal shape of the surface of the system, it is necessary in the Eq. (10) to enter the expression for the surface area and volume nanoforms. To implement this approach, it is necessary to take advantage of the fundamental dependencies between the parameters, characteristic of fractal approximations:

$$
S=N_{s} \cdot r^{D_{o s}} \text { and } V=N_{V} \cdot r^{D_{0 v}}
$$

where, $N_{s}$ and $N_{V}$ are constant proportionality factors depending on the geometry of the system taking into account the fractal dimension of the described parameters.

Then, forming the differentials for the area and volumes of fractal surfaces in accordance with Eq. (10), we obtain the following expression for the surface components of the phase chemical potential:

$$
\mu_{S}=\frac{\alpha M}{\rho} \cdot \frac{N_{s} D_{0 s}}{N_{v} D_{0 v}} \cdot r^{D_{0 s}-D_{0 v}}
$$

where, $r$ is the characteristic (length) size of the analyzed space.

The obtained expression is used to estimate the magnitude of the surface energy of the thin DLC film deposited on Ge substrate. As the input data to perform calculations, AFM image is used.

\section{Experimental Results and Numerical Calculation}

For more specific investigation, we examined DLC layers deposited by CVD method on Ge substrate. Films were deposited from dc-plasma excited by the vapor of mixture of carrying gases and hydrocarbon 
(in our case toluene $\mathrm{C}_{7} \mathrm{H}_{8}$ ) in crossed electric and magnetic fields. In the technological process ion and magnetron sources (or both of them simultaneously) were used [17-20].

It is possible to study the surface transformation of these nanolayers upon deposition time by examining them with AFM system Solver Nano-NT-MDT (Fig. 2). Also, it is possible to sketch out their boundary line (Fig. 3) which enables one to draw an idea of peculiarities of formation these layers.

During the initial stage of the formation of layers, granular structures (granules) are formed, which combine to form granules of the same type, but already with large sizes and so on. The granules are shown in Fig. 3. When these granules begin to overlap each other a layer is formed. On the shaped layer, another fragmented layer is formed and so on. From Figs. 4(a) and 4(b) the cross sectional analysis and histogram of the distribution of surface layer thickness, are shown respectively. Particularly, in Fig. 4(a), the approximate trajectory of the probe is shown on the surface of the film, and the thicknesses of the upper two layers of the film are also shown. In Fig. 4(b), we see that the peak of the thickness distribution of the surface layer is approximately $0.35 \mathrm{~nm}$. Analysis reveal the topmost fragmented islet-like layers have thickness of about $0.3 \mathrm{~nm}$, which indicates that an atomic layer (the thickness of the epitaxial grapheme is $0.33 \mathrm{~nm}$ ) of carbon exists there. These results, as

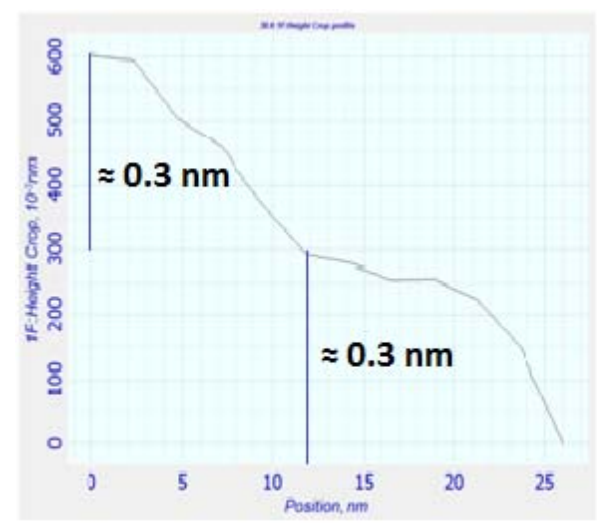

(a) line scan well as the cross-sectional and statistical analysis allow us to state that our layers can be subjected to fractal analysis.

Applying already considered to be classical mathematical formalism of multifractal analysis together with computational programs the fractal parameters are deduced which makes it possible to present some specific physical properties, in particular the surface energy of the layer, as a function of these parameters. Accordingly, the Regne's fractal coefficient decreases with the deposition time, which

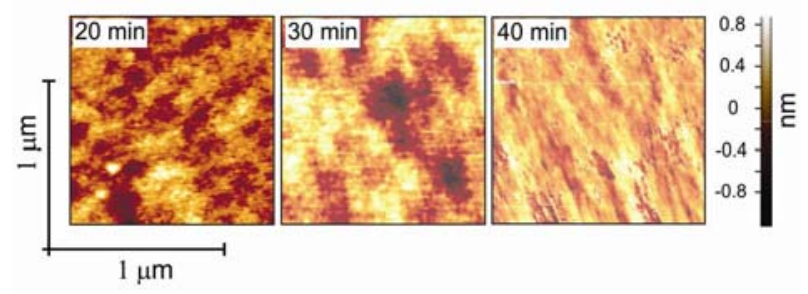

Fig. 2 AFM images of DLC samples with different CVD deposition time.

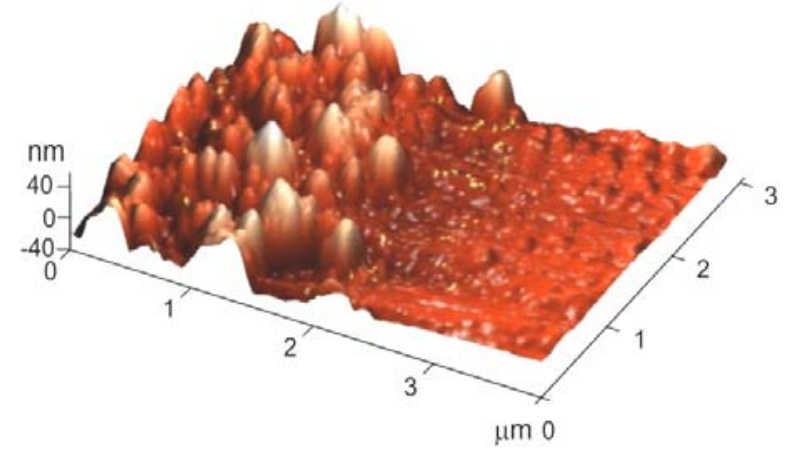

Fig. 3 The border line between Ge substrate and DLC film.

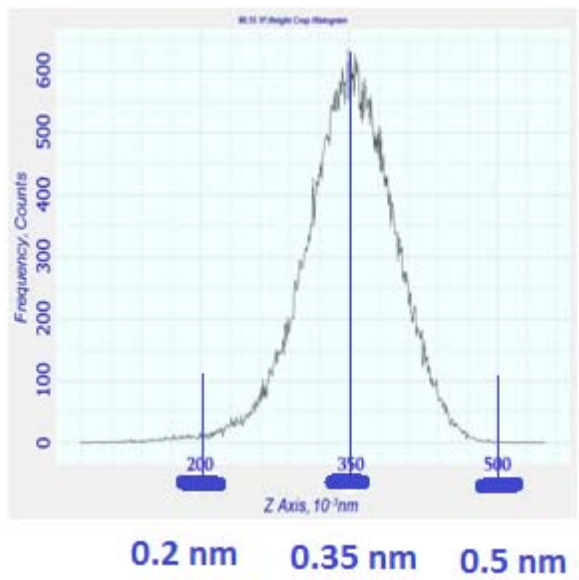

(b) histogram

Fig. 4 (a) Cross sectional analysis of DLC surface, (b) histogram of surface. 
Table 1 Surface and volume Regne's coefficients for different deposition time values.

\begin{tabular}{lllll}
\hline & $10 \mathrm{~min}$ & $20 \mathrm{~min}$ & $30 \mathrm{~min}$ & $40 \mathrm{~min}$ \\
\hline$D_{0 S}$ & 2.75 & 2.64 & 2.54 & 2.5 \\
$D_{0 \mathrm{~V}}$ & 2.90 & 2.88 & 2.86 & 2.86 \\
\hline
\end{tabular}

is the result of fact that the surface becomes more even and ordered. Software was developed by us to calculate Regne coefficients for surface and the bulk. Thus, obtained for $10 \mathrm{~min}, 20 \mathrm{~min}, 30 \mathrm{~min}$ and $40 \mathrm{~min}$ deposition time Regne's fractal coefficients $D_{0 S}$ and $D_{0 V}$ are shown in Table 1.

Introducing these values into Eq. (12) we obtained the following relations for surface energy:

$$
\mu_{S 1}: \mu_{S 2}: \mu_{S 3}: \mu_{S 4}=1: 1.49: 2.15: 2.32
$$

On the other hand, similar relation for surface potential can be obtained also by using data measured by Kelvin probe method. The Kelvin probe technique relies on the detection of an electric field between a sample material and probe material. The electric field can be varied by the voltage that is applied to the sample relative to the probe. Although the Kelvin probe technique only measures a work function difference, it is possible to obtain an absolute work function by first calibrating the probe against a reference material (with known work function) and then using the same probe to measure a desired sample. The Kelvin probe technique is used to obtain work function maps of a surface with extremely high spatial resolution, by using a sharp tip for the probe in the semi contacting AFM mod.

$$
\mu_{S 1}: \mu_{S 2}: \mu_{S 3}: \mu_{S 4}=100: 150: 225: 250
$$

Summarizing the calculations, it turns out that the numerical results obtained by the method of multifractal analysis Eq. (13) and measurements Eq. (14) are very similar.

\section{Conclusions}

In summary, it can be argued, that DLC film as a self-organized system can be analyzed by the method of multifractal analysis, because in the process of forming of the DLC film some different fractal regularities are maintained. Range parameter decreases with increasing deposition time for surface, while for volume it is almost unchanged. It suggests that fractal regularity during the formation of the film surface keeps constantly changing (and most of surface properties are changing with it), while during the formation of the whole volume of the film it is almost unchanged. Investigation made by multifractal analysis method gives similar results with experimental data. Thus, it can be said that the surface potential and the physical properties associated with it are closely related to the fractal principle of film and its surface formation. And secondly, from the results of the analysis, it can be stated that on the surface of the fractal layer of the obtained DLC, the carbon layers of the atomic thickness, which are responsible for the surface properties of the film.

\section{Acknowledgments}

The author wishes to express his appreciation to head of laboratory "Heliotechnic" Prof. Zhozef Panosyan and senior researcher Mr. Serjik Voskanyan for their useful tips and careful attention during the study.

\section{References}

[1] Sun, X., Fu, Z., and Wu, Z. 2002. "Fractal Processing of AFM Images of Rough ZnO Films." Materials Characterization 48: 169-75.

[2] Kurt Yücel, H., Kurt, E., and Salamov, B. G. 2004. "Fractal Processing for an Analysis of the Quality and Resistivity of Large Semiconductor Plates." Cryst. Res. Technol. 39(9): 743-53.

[3] Fang, T. H., Jian, S. R., and Chuu, D. S. 2003. "Nanotribology and Fractal Analysis of ZnO Thin Films Using Scanning Probe Microscopy.” J. Phys. 36: 878-83.

[4] Jafari, G. R., Fazeli, S. M., Ghasemi F., and Kavei, G. 2003. "Stochastic Analysis and Regeneration of Rough Surface.” Phys. Rev. Lett. 91 (22): 226101-6.

[5] Rerkowitz, B., and Hadad A. 1997. "Fractal and Multifractal Measures of Natural and Synthetic Fracture Networks.” J. Geophys. Research 102 (B6): 12205-18. 
[6] Bulashenko, O. M., Luo, K. J., Grahn, H. T., Ploog, K. H., and Bonilla, L. L. 1999. "Multifractal Dimension of Chaotic Attractors in a Driven Semiconductor Superlattice." Phys. Rev. B 60 (8): 5694-97.

[7] Yadav, R. P., Singh, U. B., Mittal, A. K., and Dwivedi, S. 2014. "Investigating the Nanostructured Gold Thin Film Using the Multifractal Analysis." Appl. Phys. A 117 (4): 2159-66.

[8] Moskvin, P., Kryzhanivskyy, V., Rashkovetskyi, L., Lytvyn, P., and Vuichyk, M. 2014. "Multifractal Analysis of Areas of Spatial Forms on Surface of ZnxCd1-xTe-Si (111) Heterocompositions." Journal of Crystal Growth 404: 204-9.

[9] Snopok, B., Strizhak, P., Kostyukevich, E. V., and Venger, E. F. 1999. "Interfacial Architecture on the Fractal Support: Polycrystalline Gold Films as Support of Self-Assembling Layers." Semiconductor Physics, Quantum Electronics \& Optoelectronics 2 (3): 86-97.

[10] Talu, S., Markovic, Z., Stach, S., Marković, B. T., and Țălu, M. 2014. "Multifractal Characterization of Single Wall Carbon Nanotube Thin Films Surface Upon Exposure to Optical Parametric Oscillator Laser Irradiation." Applied Surface Science 289: 97-107.

[11] Miller, J. D., Veeramasuneni, S., Drelich, J., Yalamanchili, M. R., and Yamauchi, G. 1996. "Effect of Roughness as Determined by Atomic Force Microscopy on the Wetting Properties of PTFE Thin Films." Polymer Engineering and Science 36 (14): 1849-55.

[12] Raoufi, D., Fallah, H. R., Kiasatpour, A., and Rozatian, A. S. H. 2008. "Multifractal Analysis of ITO Thin Films Prepared by Electron Beam Method." Applied Surface Science 254: 2168-73.

[13] Fang, T. H., and Chang, W. J. 2003. "Effects of AFN-Based Nanomachining Process on Aluminum
Surface." Journal of Physics and Chemistry of Solids 64: 913-8.

[14] Altaisky, M. V., Chernenko, L. P., Balebanov, V. M., Erokhin, N. S., and Moiseev, S. S. 2000. "Multifractal Analysis of AFM Images of Nb Thin Film Surfaces." Particles and Nuclei, Letters 99 (2): 14-26.

[15] Talu, S., Stach, S., Mahajan, A., and Talu, M. 2014. "Multifractal Characterization of Water Soluble Copper Phthalocyanine Based Films Surface." Electron. Mater. Lett. 10 (4): 1-12.

[16] Arneodo, A., Decoster, N., and Roux, S. G. 2000. “A Wavelet-Based Method for Multifractal Image Analysis. Methodology and Test Applications on Isotropic and Anisotropic Random Rough Surfaces.” Eur. Phys. J. B 15: 567-600.

[17] Panosyan, Z. R., Darbasyan, A. T., Voskanyan, S. S., and Yengibaryan, Y. V. 2014. "Methods for Preparation of Transparent Conductive Diamond-Like Carbon Films Mechanisms of Conductivity Formation." Journal of Contemporary Physics 49 (6): 286-92.

[18] Gharibyan, A., and Hayrapetyan, D. 2010. "Preparation and Investigation of Diamond-Like Carbon Nanocomposite Thin Films for Nanophotonics." In Proceedings of SPIE, NANOPHOTONIC Materials VII. 7755 (1): 279-84.

[19] Gharibyan, A, Hayrapetyan, D., Panosyan, Zh., et al. 2011. "Preparation and Investigation of Diamond-Like Carbon Nanustructured Films." Optical Society of America, Applied Optics 50 (31).

[20] Panosyan, Z. R., Sharps, P. R., et al. 2015. "Growig, Physical Properties Study and Application of Nanostructure Carbon Multifunctional Films." In Proceedings of THE Ninth International Conference Semiconductor Micro- and Nanoelectronics. 